\title{
ANTIOXIDANTIEN ALS STRATEGIE ZUR OPTIMIERUNG DER KRYO- KONSERVIERUNG VON STAMMZELLEN FÜR DAS TISSUE ENGINEERING
}

\author{
L. Smits ${ }^{1}$, N. Hofmann ${ }^{1}$, B. Glasmacher ${ }^{1}$ \\ ${ }^{1}$ Institut für Mehrphasenprozesse (IMP), Leibniz Universität Hannover, Deutschland \\ smitsli@stud.uni-hannover.de
}

\begin{abstract}
Kontrolliertes Einfrieren in den Temperaturbereich zwischen $-80^{\circ} \mathrm{C}$ und $-196{ }^{\circ} \mathrm{C}$ erlaubt es Zellen, bei gleichzeitigem Erhalt ihrer Vitalität nach dem Auftauprozess, langfristig zu lagern. Durch die Verwendung von Antioxidantien sollte in der vorliegenden Arbeit eine Steigerung der Rekultivierbarkeit von mesenchymalen Stammzellen nach der Kryokonservierung erzielt werden. Der Zusatz von $100 \mu \mathrm{M} \alpha-$ Tocopherol steigerte die Rekultivierungseffzienz der Stammzellen um $117,5 \%$ gegenüber der Kontrolle ohne Antioxidans. Darüber hinaus konnte gezeigt werden, dass eine Reduzierung der DMSO-Konzentration möglich ist, da Versuche mit $200 \mu M$ a-Tocopherol und $2,5 \%$ bzw. $5 \%$ DMSO vergleichbare Resultate von ca. $164 \%$ bzw. $170 \%$ Rekultivierbarkeit erzielten.
\end{abstract}

Keywords: Kryokonservierung, Antioxidantien, Kryoadditiv, Stammzellen, $\alpha$-Tocopherol, L-Ascorbinsäure

\section{Einleitung}

Die Kryokonservierung erlaubt eine Langzeitlagerung von strukturell intakten, lebenden Zellen sowie nativem oder gezüchtetem Gewebe [1]. Eine erfolgreiche Kryokonservierung kann jedoch nur durch Zugabe von Kryoprotektiva wie z.B. Dimethylsulfoxid (DMSO) realisiert werden. Der schützenden Wirkung von DMSO während des Einfrierprozesses steht dabei die Zelltoxizität dieses Stoffes gegenüber [2]. Aus diesem Grund wird an alternativen, weniger zellbelastenden Gefrierschutzstrategien mit neuen effektiven Additiven geforscht [3]. In diesem Zusammenhang stellt der Zusatz von Antioxidantien (AO) eine vielversprechende Möglichkeit dar, da während des Gefrier- und Auftauprozesses freie Radikale entstehen, die einen großen Anteil an Zellschädigungsereignissen haben. Der Einsatz von AO wie a-Tocopherol und L-Ascorbinsäure erhöht daher den Schutz der Zelle und verbessert die Zellvitalität nach der Kryokonservierung [4, 5]. Das lipophile, an der Zellmembran lokalisierte $\alpha$-Tocopherol ist in der Lage, Lipidoxidationsprodukte zu eliminieren und einen Abbruch der radikalischen Kettenreaktion zu bewirken. Dadurch sollte auch eine Reduktion des Kryoprotektivs DMSO möglich werden.

\section{Methoden}

Versuchsansätze: Bei den verwendeten Zellen handelte es sich um mesenchymale Stammzellen des Weißbüschelaffens Callithrix jacchus (cjMSC- FC 59). In einer vorangegangenen Studie wurde ein optimiertes Kryokonservierungsprotokoll mit dem herkömmli- chen Kryoprotektiv DMSO für diesen Zelltyp erarbeitet [6]. Bei den durchgeführten Versuchen wurde die Art und Konzentration der AO sowie die zusätzlich zugefügte Menge DMSO variiert (Tab. 1). Pro Versuchsansatz wurden $3,6 \times 10^{6}$ Zellen eingesetzt.

Tabelle 1: Getestete Stoffmengenkonzentrationen von L-Ascorbinsäure (AA) und a-Tocopherol (toc).

\begin{tabular}{c|c|c|c} 
Ansatz & DMSO $[\mathbf{v} / \mathbf{v} \%]$ & toc $[\boldsymbol{\mu M}]$ & AA $[\boldsymbol{\mu M}]$ \\
\hline 1 & 2,5 & 0 & 0 \\
\hline 2 & 5 & 0 & 0 \\
\hline 3 & ohne & $100,200,500$ & 0 \\
\hline 4 & ohne & 0 & $50,100,250$ \\
\hline 5 & 2,5 & $100,200,500$ & 0 \\
\hline 6 & 2,5 & 0 & $50,100,250$ \\
\hline 7 & 5 & $100,200,500$ & 0 \\
\hline 8 & 5 & 0 & $50,100,250$
\end{tabular}

Kryokonservierung: Die Zellen wurden mit Hilfe des am IMP entwickelten $\mu$-Freezer standardisiert eingefroren, wodurch eine gleichbleibende Qualität des Einfrierprozesses und damit die Vergleichbarkeit der Ergebnisse verschiedener Versuchsdurchläufe gewährleistet war [6]. Aufgrund der zelltoxischen Wirkung von DMSO muss das Kryoprotektiv nach dem Auftauen ausgewaschen werden. Unmittelbar danach wurde die erste Zellzahlbestimmung durchgeführt. Die restlichen Zellen wurden anschließend für $23 \mathrm{~h}$ im Inkubator bei $37^{\circ} \mathrm{C}$ und $5 \% \mathrm{CO}_{2}$ rekultiviert.

Zellvitalitätstest: Die Anzahl der Zellen in den jeweiligen Proben wurde mittels Trypanblau-Färbung bestimmt. Nach der Rekultivierung wurde zusätzlich zu den adhärenten Zellen auch die Anzahl der Zellen im Medienüberstand bestimmt. Da nur vitale Zellen die Fähigkeit besitzen an Matrizen zu adhärieren, wurden alle Zellen vom Flaschenboden als lebend und die Zellen des Überstandes als letal geschädigt betrachtet. Die Anzahl der adhärenten, vitalen Zellen wurde zur anfänglich eingesetzten Zellzahl ins Verhältnis gesetzt, dies beschreibt die Rekultivierungseffzienz (RE).

\section{Ergebnisse}

Kryokonservierung ohne Kryoprotektiv: Abbildung 1a zeigt Zellen nach Kryokonservierung ohne DMSO. Im Gegensatz zu den mit Kryoprotektiv eingefrorenen Zellen (Abb. 1b) sind hierbei nach der Rekultivierung über $90 \%$ der Zellen letal geschädigt und nicht fähig an der Matrix zu adhärieren. 


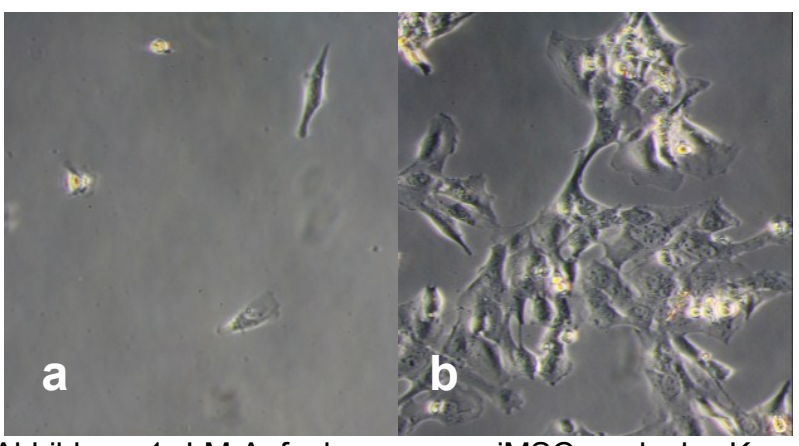

Abbildung 1: LM-Aufnahmen von cjMSC nach der Kryokonservierung ohne (a) und mit Kryoprotektiv (b) (200x).

Rekultivierungseffizienz: Der Anteil rekultivierbarer Zellen ist bei Verwendung von a-Tocopherol (toc) gegenüber den Kontrollansätzen, aber auch gegenüber den Ansätzen mit L-Ascorbinsäure $(\mathrm{AA})$ deutlich höher (Abb. 2).

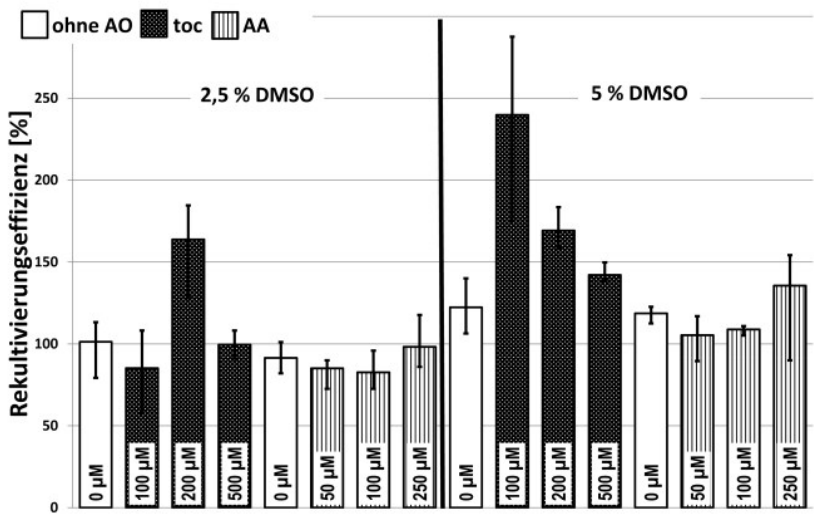

Abbildung 2: Anteil vitaler Zellen in Bezug auf die Gesamtzellzahl vor der Kryokonservierung in [\%] $(n=4)$.

Die prozentualen Anteile rekultivierbarer Zellen liegen bei Versuchen mit toc sowohl mit 2,5\%, als auch mit $5 \%$ DMSO deutlich höher, als die der Ansätze ohne AO-Zusatz. Die Differenz beträgt $62,5 \%$ für $2,5 \%$ DMSO mit $200 \mu \mathrm{M}$ toc und bei Verwendung von $5 \%$ DMSO mit $100 \mu \mathrm{M}$ toc sogar $117,5 \%$. Beim Zusatz von $250 \mu \mathrm{M}$ AA liegt die Differenz dagegen nur bei $6,7 \%$ bzw. 16,9\%. Die übrigen Versuche mit AA-Zusatz zeigen schlechtere Ergebnisse im Vergleich zu den Standardansätzen. Generell weisen jedoch Ansätze mit AO-Zusatz höhere Rekultivierungseffizienzen auf, wobei sich die Differenz von Kontrollansatz zu behandeltem Ansatz mit steigender DMSO-Konzentration vergrößert. Zusätzlich wird die Kryoprotektivreduzierung durch AO-Zugabe kompensiert. Versuchsansätze mit $200 \mu \mathrm{M}$ toc und $2,5 \%$ bzw. $5 \%$ DMSO erzielten vergleichbare Resultate von $163,8 \%$ bzw. 169,2 \% bei der Rekultivierbarkeit.

\section{Diskussion}

Eine erfolgreiche Kryokonservierung ist unter anderem durch die Wahl einer optimalen Kühl- bzw. Erwärmungsrate und den Einsatz eines geeigneten
Kryoprotektivs realisierbar $[1,7,9]$. Die Ausbeute an strukturell intakten, vitalen Zellen kann zusätzlich durch die Kompensation des während der Kryokonservierung auftretenden oxidativen Stresses weiter gesteigert werden [4]. Dieser entsteht in der Regel innerhalb der Zelle durch Belastung mit reaktiven Sauerstoffspezies bei gleichzeitig nicht ausreichender Zellabwehr gegen anfallende Radikale [8]. Der Einsatz von Antioxidantien, welche den Abbruch radikalischer Kettenreaktionen bewirken, sollte demzufolge eine Erhöhung der Zellvitalität hervorrufen.

Durch die vorliegende Studie konnte gezeigt werden, dass Antioxidantien positive Effekte auf die Proliferation der Primatenstammzellen nach der Kryokonservierung ausüben. Besonders deutlich ist die Steigerung der Rekultivierungseffizienz, die durch a-Tocopherol hervorgerufen wurde. Das an der Zellmembran lokalisierte Antioxidans eliminiert Lipidoxidationsprodukte und sorgt damit für einen Kettenabbruch der zellschädigenden Reaktion [5]. Die niedrigere Effizienz der Rekultivierungsergebnisse beim Einsatz von LAscorbinsäure könnte daraus resultieren, dass dieses Antioxidans gleichzeitig auch als Prooxidans wirken und destruktive Radikalreaktionen eingehen kann [8]. Weiterhin wurde deutlich, dass trotz Reduzierung der Kryoprotektivkonzentration eine vergleichbare Rekultivierungseffizienz erzielt wurde. Da sich durch die Zugabe der Antioxidantien der absolute Anteil des zelltoxischen Kryoprotektivs verringert, wird zusätzlich die schädliche Belastung bei Raumtemperatur insgesamt herabgesetzt. In zukünftigen Untersuchungen soll nun geprüft werden, ob die Kombination beider Stoffe und der Zusatz weiterer alternativer Kryoprotektiva die Rekultivierungseffizienz nach der Kryokonservierung zusätzlich steigern kann und damit ein vollständiger Ersatz des toxischen DMSO ermöglicht wird.

\section{Danksagung}

Das Projekt wurde finanziell durch das Cluster of Excellence REBIRTH unterstützt (EXC 62/1).

\section{Literatur}

[1] Day, J et al.: Methods Mol Biol. 368: 141-51 (2007)

[2] Pegg, D et al.: Semin Reprod Med. 20(1): 5-13 (2002)

[3] Sun, $\mathrm{H}$ et al.: CryoLetters 3 (6): 485-93 (2012)

[4] Uchendu, E et al.: Plant Cell Rep. 29(1): 25-35 (2010)

[5] Packer, L et al.: Nutrition 131(2): 369-73 (2001)

[6] Hofmann, $\mathrm{N}$ et al.: $D K V$, ISBN 978-3-932715-47-1 (2011)

[7] Mazur, P et al.: Exp Cell Res. 71(2): 345-55 (1972)

[8] Monteiro, J et al.: Reprod Domest Anim. 44 Suppl 2: 359-362 (2009)

[9] Hofmann, N et al.: Problems Cryobiol. 21(4): 35363 (2011) 